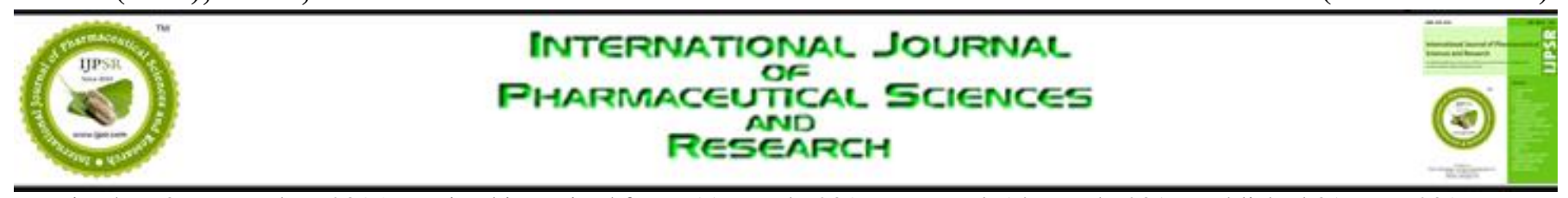

Received on 07 December, 2015; received in revised form, 11 March, 2016; accepted, 14 March, 2016; published 01 June, 2016

\title{
TRANSDERMAL DRUG DELIVERY SYSTEM: A REVIEW
}

\author{
Himanshi Tanwar * and Ruchika Sachdeva \\ Guru Jambheshwar University of Science \& Technology, Hisar, Haryana, India.
}

Keywords:

Topical, Reservoir,

Transdermal, Epidermis

\section{Correspondence to Author: \\ Himanshi Tanwar}

Guru Jambheshwar

University of Science \& Technology,

Hisar, Haryana, India.

Email: tanwarhimanshi@gmail.com

\begin{abstract}
A transdermal patch is a medicated adhesive patch that is placed on the skin to deliver a specific dose of medication through the skin and into the bloodstream. Often, this promotes healing to an injured area of the body. An advantage of a transdermal drug delivery route over other types of medication delivery such as oral, topical, intravenous, intramuscular, etc. is that the patch provides a controlled release of the medication into the patient, usually through either a porous membrane covering a reservoir of medication or through body heat melting thin layers of medication embedded in the adhesive. Transdermal drug delivery offers controlled release of the drug into the patient, it enables a steady blood level profile, resulting in reduced systemic side effects and, sometimes, improved efficacy over other dosage forms. The main objective of transdermal drug delivery system is to deliver drugs into systemic circulation through skin at predetermined rate with minimal inter and intrapatient variations.
\end{abstract}

INTRODUCTION: During the past few years, interest in the development of novel drug delivery systems for existing drug molecules has been renewed. The development of a novel delivery system for existing drug molecules not only improves the drug's performance in terms of efficacy and safety but also improves patient compliance and overall therapeutic benefit to a significant extent. ${ }^{1}$ Transdermal Drug Delivery System (TDDS) are defined as self contained, discrete dosage forms which are also known as "patches" ${ }^{2,3}$ when patches are applied to the intact skin, deliver the drug through the skin at a controlled rate to the systemic circulation. ${ }^{4}$ TDDS are dosage forms designed to deliver a therapeutically effective amount of drug across a patient's skin. ${ }^{5}$

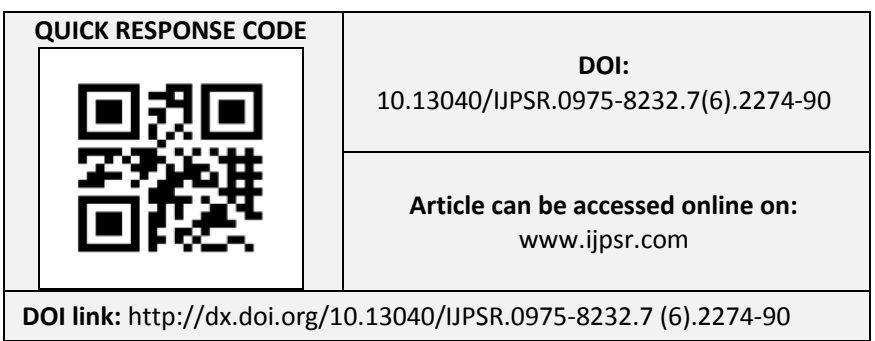

The main objective of transdermal drug delivery system is to deliver drugs into systemic circulation into the skin through skin at predetermined rate with minimal inter and intra patient variation. ${ }^{3}$ Currently transdermal delivery is one of the most promising methods for drug application. ${ }^{6}$ It reduces the load that the oral route commonly places on the digestive tract and liver. It enhances patient compliances and minimizes harmful side effects of a drug caused from temporary over dose and is convenience in transdermal delivered drugs that require only once weakly application. ${ }^{7}$

That will improves bioavailability, more uniform plasma levels, longer duration of action resulting in a reduction in dosing frequency, reduced side effects and improved therapy due to maintenance of plasma levels up to the end of the dosing interval compared to a decline in plasma levels with conventional oral dosage forms. ${ }^{8}$ Transdermal delivery not only provides controlled, constant administration of drugs, but also allows continuous input of drugs with short biological half lives and eliminates pulsed entry into systemic circulation, 
which often causes undesirable side effects. ${ }^{3}$ Several important advantages of transdermal drug delivery are limitations of hepatic first pass metabolism, enhancement of therapeutic efficacy and maintenance of steady plasma level of drug. ${ }^{1}$ The developments of TDDS is a multidisciplinary activity that encompasses fundamental feasibility studies starting from the selection of drug molecule to the demonstration of sufficient drug flux in an $e x$ vivo and in vivo model followed by fabrication of a drug delivery system that meets all the stringent needs that are specific to the drug molecule (physicochemical, stability factors), the patient (comfort and cosmetic appeal), the manufacturer (scale up and manufacturability) and most important economy. ${ }^{7}$

The first transdermal system, Transderm SCOP was approved by FDA in 1979 for the prevention of nausea and vomiting associated with travel. Most transdermal patches are designed to release the active ingredient at a zero order rate for a period of several hours to days following application to the skin. This is especially advantageous for prophylactic therapy in chronic conditions. ${ }^{9}$ The evidence of percutaneous drug absorption may be found through measurable blood levels of the drug, detectable excretion of the drug and its metabolites in the urine and through the clinical response of the patient to the administered drug therapy. ${ }^{10}$

\section{Transdermal route and drug delivery prospects Skin:}

\section{The largest organ:}

The skin is the largest organ of the human body which covers a surface area of approximately 2 sq.m. and receives about one third of the blood circulation through the body. ${ }^{5}$ It serves as a permeability barrier against the transdermal absorption of various chemical and biological agents. It is one of the most readily available organs of the body with a thickness of few millimeters $(2.970 .28 \mathrm{~mm})$ which,

- Separates the underlying blood circulation network from the outside environment.

- Serves as a barrier against physical, chemical and microbiological attacks.
- Acts as a thermostat in maintaining body temperature.

- Plays role in the regulation of blood pressure.

- Protects against the penetration of UV rays.

- Skin is a major factor in determining the various drug delivery aspects like permeation and absorption of drug across the dermis. The diffusional resistance of the skin is greatly dependent on its anatomy and ultrastructure. ${ }^{12}$

\section{Anatomy of Skin:}

The structure of human skin (fig.1) can be categorized into four main layers:

- The epidermis

$\checkmark$ The viable epidermis

\section{A non-viable epidermis (Stratum corneum)}

- The overlying dermis

The innermost subcutaneous fat layer (Hypodermis) ${ }^{13}$

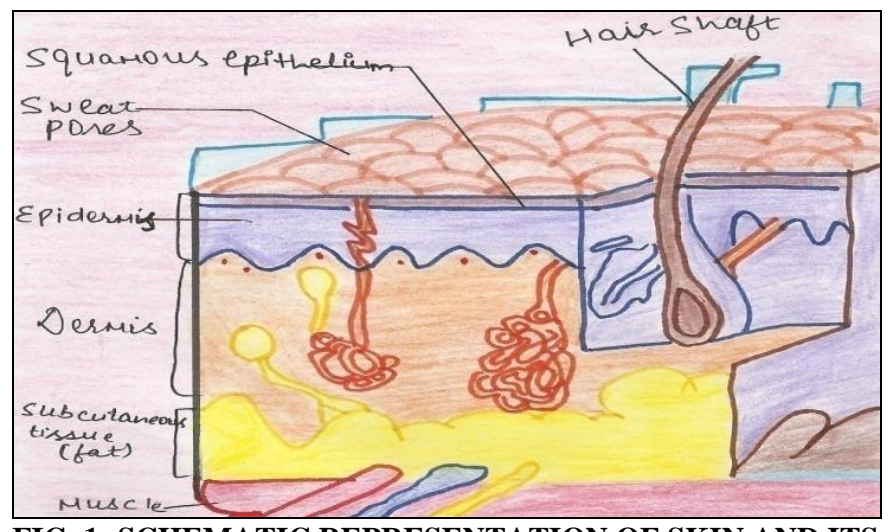

FIG. 1: SCHEMATIC REPRESENTATION OF SKIN AND ITS APPENDAGES

\section{The Epidermis:}

The epidermis is a continually self-renewing, stratified squamous epithelium covering the entire outer surface of the body and primarily composed of two parts: the living or viable cells of the malpighian layer (viable epidermis) and the dead cells of the stratum corneum commonly referred to as the horny layer ${ }^{5}$. Viable epidermis is further 
classified into four distinct layers as shown in Fig. $\mathbf{2}^{12}$

- Stratum lucidum

- Stratum granulosum

- Stratum spinosum

- Stratum basale

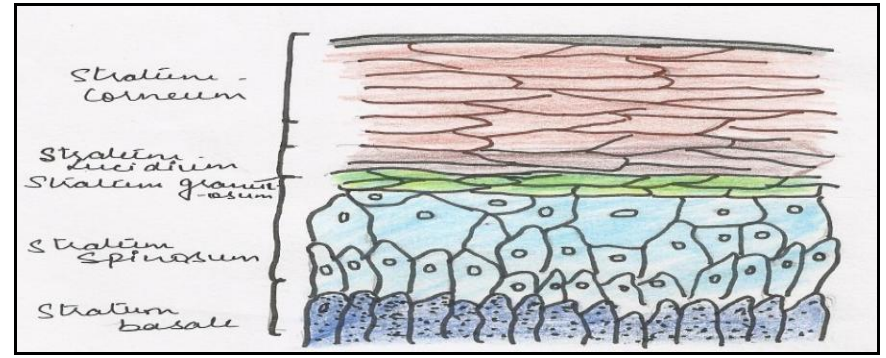

FIG. 2: SCHEMATIC REPRESENTATION OF ANATOMY OF EPIDERMIS

\section{Stratum corneum:}

This is the outermost layer of skin also called as horny layer. It is the rate limiting barrier that restricts the inward and outward movement of chemical substances. The barrier nature of the horny layer depends critically on its constituents: $75-80 \%$ proteins, $5-15 \%$ lipids, and $5-10 \%$ ondansetron material on a dry weight basis.

Stratum corneum is approximately $10 \mathrm{~mm}$ thick when dry but swells to several times when fully hydrated. It is flexible but relatively impermeable. The architecture of horny layer (figure 3 ) may be modeled as a wall-like structure with protein bricks and lipid mortar. It consists of horny skin cells (corneocytes) which are connected via desmosomes (protein-rich appendages of the cell membrane). The corneocytes are embedded in a lipid matrix which plays a significant role in determining the permeability of substance across the skin. ${ }^{11}$

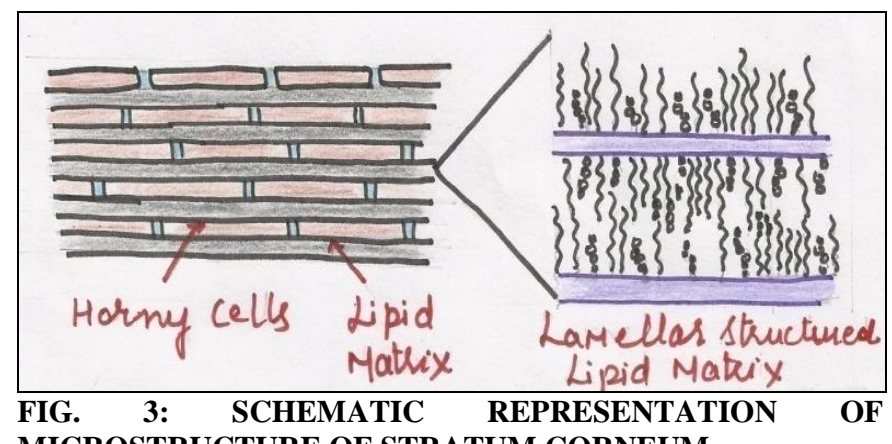

Viable epidermis:

This is situated beneath the stratum corneum and varies in thickness from $0.06 \mathrm{~mm}$ on the eyelids to $0.8 \mathrm{~mm}$ on the palms. Going inwards, it consists of various layers as stratum lucidum, stratum granulosum, stratum spinosum, and the stratum basale. In the basale layer, mitosis of the cells constantly renews the epidermis and this proliferation compensates the loss of dead horny cells from the skin surface. As the cells produced by the basale layer move outward, they itself alter morphologically and histochemically, undergoing keratinization to form the outermost layer of stratum corneum. ${ }^{14}$ shown in Fig. 4.

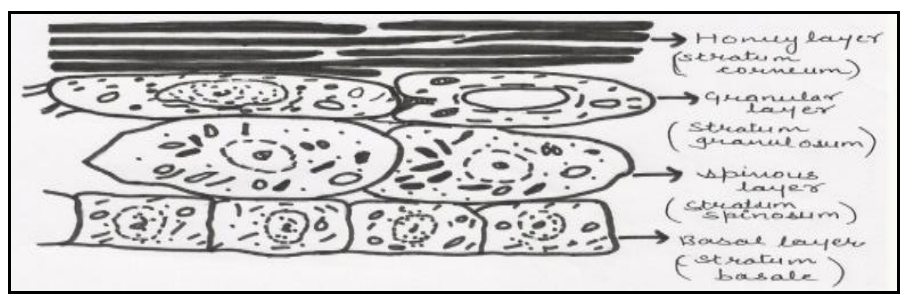

FIG. 4: SCHEMATIC REPRESENTATION OF DIFFERENT LAYERS OF EPIDERMIS

\section{Dermis:}

Dermis is the layer of skin just beneath the epidermis which is 3 to $5 \mathrm{~mm}$ thick layer and is composed of a matrix of connective tissues, which contains blood vessels, lymph vessels, and nerves. The cutaneous blood supply has essential function in regulation of body temperature. It also provides nutrients and oxygen to the skin, while removing toxins and waste products. Capillaries reach to within $0.2 \mathrm{~mm}$ of skin surface and provide sink conditions for most molecules penetrating the skin barrier. The blood supply thus keeps the dermal concentration of permeate very low, and the resulting concentration difference across the epidermis provides the essential driving force for transdermal permeation. In terms of transdermal drug delivery, this layer is often viewed as essentially gelled water, and thus provides a minimal barrier to the delivery of most polar drugs, although the dermal barrier may be significant when delivering highly lipophillic molecules. ${ }^{13}$

\section{Hypodermis:}

The hypodermis or subcutaneous fat tissue supports the dermis and epidermis. It serves as a fat storage area. This layer helps to regulate temperature, provides nutritional support and mechanical 
protection. It carries principal blood vessels and nerves to skin and may contain sensory pressure organs. For transdermal drug delivery, drug has to penetrate through all three layers and reach in systemic circulation. ${ }^{14}$

\section{Percutaneous absorption:}

Before a topically applied drug can act either locally or systemically, it must penetrate through stratum corneum. Percutaneous absorption is defined as penetration of substances into various layers of skin and permeation across the skin into systemic circulation. ${ }^{11}$ Percutaneous absorption of drug molecules is of particular importance in transdermal drug delivery system because the drug has to be absorbed to an adequate extent and rate to achieve and maintain uniform, systemic, therapeutic levels throughout the duration of use. In general once drug molecule cross the stratum corneal barrier, passage into deeper dermal layers and systemic uptake occurs relatively quickly and easily. ${ }^{9}$

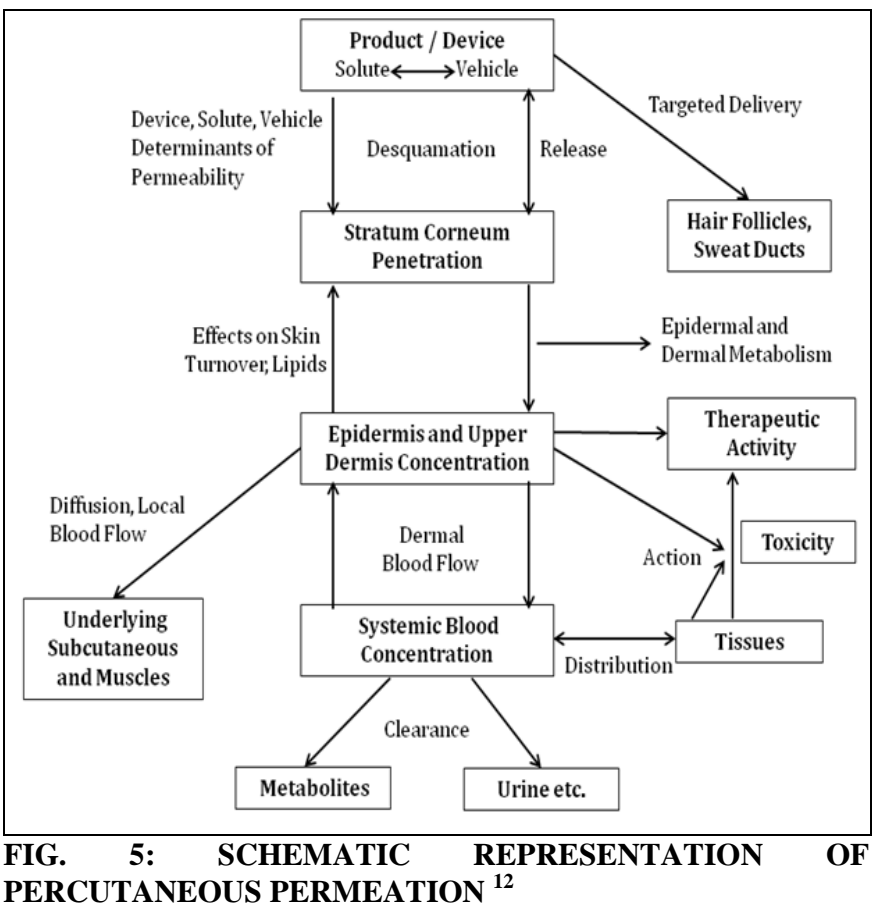

The release of a therapeutic agent from a formulation applied to the skin surface and its transport to the systemic circulation is a multistep process (figure 5) which involves:

- Dissolution within and release from the formulation
- Partitioning into the skin's outermost layer, the stratum corneum (SC)

- Diffusion through the SC, principally via a lipidic intercellular pathway.

- Partitioning from the SC into the aqueous viable epidermis, diffusion through the viable epidermis and into the upper dermis, uptake into the papillary dermis (capillary system) and into the microcirculation. ${ }^{15}$

\section{Routes of drug penetration through skin:}

In the process of percutaneous permeation, a drug molecule may pass through the epidermis itself or may get diffuse through shunts, particularly those offered by the relatively widely distributed hair follicles and eccrine glands as shown in figure 6 . In the initial transient diffusion stage, drug molecules may penetrate the skin along the hair follicles or sweat ducts and then absorbed through the follicular epithelium and the sebaceous glands. When a steady state has been reached the diffusion through the intact Stratum corneum becomes the primary pathway for transdermal permeation ${ }^{11}$

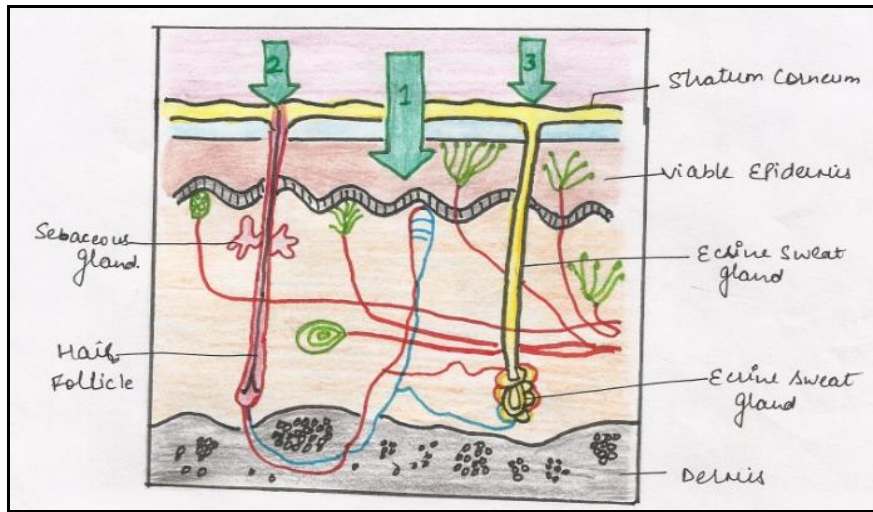

FIG. 6: POSSIBLE MACRO ROUTES FOR DRUG PENETRATION 1) INTACT HORNY LAYER, 2) HAIR FOLLICLES AND 3) ECCRINE SWEAT GLANDS

For any molecules applied to the skin, two main routes of skin permeation can be defined:

Transepidermal route

Transfollicular route

\subsection{Transepidermal route:}

In transepidermal transport, molecules cross the intact horny layer. Two potential micro-routes of 
entry exist, the transcellular (or intracellular) and the intercellular pathway as shown in Fig. 7, 8

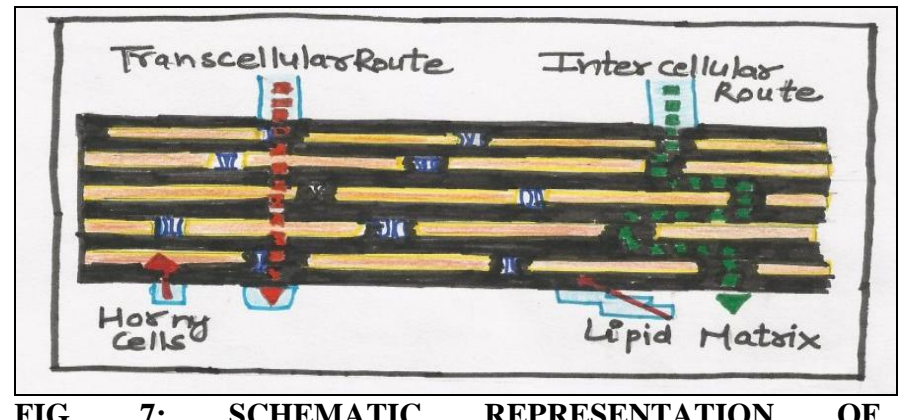

TRANSEPIDERMAL ROUTE

Both polar and non-polar substances diffuse via transcellular and intercellular routes by different mechanisms. The polar molecules mainly diffuse through the polar pathway consisting of "bound water" within the hydrated stratum corneum whereas the non-polar molecules dissolve and diffuse through the non-aqueous lipid matrix of the stratum corneum. Thus the principal pathway taken by a penetrant is decided mainly by the partition coefficient $(\log \mathrm{K})$. Hydrophilic drugs partition preferentially into the intracellular domains, whereas lipophillic permeants (octanol/water log K $>$ 2) traverse the stratum corneum via the intercellular route. Most molecules pass the stratum corneum by both routes. ${ }^{5}$

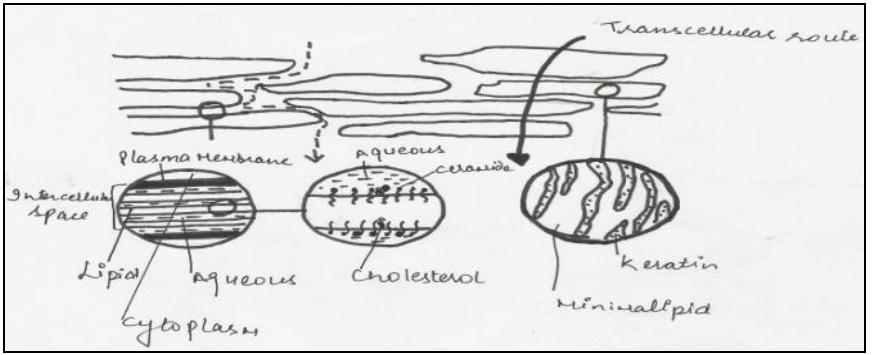

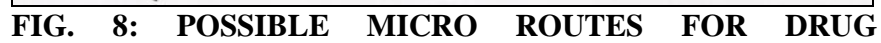
PENETRATION ACROSS HUMAN SKIN INTERCELLULAR OR TRANSCELLULAR.

\subsection{Transfollicular route (Shunt pathway):}

This route comprises transport via the sweat glands and the hair follicles with their associated sebaceous glands. Although these routes offer high permeability, they are considered to be of minor importance because of their relatively small area, approximately $0.1 \%$ area of the total skin. This route seems to be most important for ions and large polar molecules which hardly permeate through the stratum corneum $^{5}$

\section{Barrier functions of the skin:}

The top layer of skin is most important function in maintaining the effectiveness of the barrier. Here the individual cells overlie each other and are tightly packed, preventing bacteria from entry and maintaining the water holding properties of the skin $^{14}$. Stratum corneum mainly consists of the keratinized dead cell and water content is also less as compared to the other skin components. ${ }^{15}$ Lipids are secreted by the cells from the base layer of the skin to the top. These lipid molecules join up and form a tough connective network, in effect acting as the mortar between the bricks of a wall.

5 Basic Principal of Transdermal permeation: ${ }^{1,7}$ Transdermal permeation is based on passive diffusion ${ }^{1}$. Skin is the most intensive and readily accessible organ of the body as only a fraction of millimeter of tissue separates its surface from the underlying capillary network. ${ }^{7}$ The release of a therapeutic agent from a formulation applied to the skin surface and its transport to the systemic circulation is a multistep process ${ }^{1}$, which includes

1) Diffusion of drug from drug to the rate controlling membrane.

2) Dissolution within and release from the formulation.

3) Sorption by stratum corneum and penetration through viable epidermis.

4) Uptake of drug by capillary network in the dermal papillary layer.

5) Effect on the target organ.

6) Partitioning into the skin's outermost layer, the stratum corneum.

7) Diffusion through the stratum corneum, principal via a lipidic intercellular pathway.

\section{Properties that influence transdermal delivery: ${ }^{16}$}

1. Release of the medicament from the vehicle

2. Penetration through the skin barrier. 
3. Activation of the pharmacological response.

6 Advantages of transdermal drug delivery: ${ }^{(1,8,}$ $16,17,18,19)$

- Transdermal drug delivery enables the avoidance of gastrointestinal absorption with its associated pitfalls of enzymatic and $\mathrm{pH}$ associated deactivation.

- Avoidance of first pass metabolism.

- The lack of peaks in plasma concentration can reduce the risk of side effects, thus drugs that require relatively consistent plasma levels are very good candidate for transdermal drug delivery.

- As a substitute for oral route.

- The patch also permit constant dosing rather than the peaks and valley in medication level associated with orally administered medication.

- Rapid notifications of medication in the event of emergency as well as the capacity to terminate drug effects rapidly via patch removal.

- Avoidance of gastro intestinal incompatibility.

- Convenience especially notable in patches that require only once weekly application, such a simple dosing regimen can aid in patient adherence to drug therapy.

- Minimizing undesirable side effects.

- Provide utilization of drug with short biological half lives, narrow therapeutic window.

- Avoiding in drug fluctuation drug levels.

- Inter and intra patient variation.
- Termination of therapy is easy at any point of time.

- Provide suitability for self administration.

- They are non invasive, avoiding the inconvenience of parentral therapy.

- The activity of drugs having a short half life is extended through the reservoir of drug in the therapeutic delivery system and its controlled release.

- It is of great advantages in patients who are nauseated or unconscious.

- Transdermal patches are better way to deliver substances that are broken down by the stomach aids, not well absorbed from the gut, or extensively degraded by the liver.

- Transdermal patches are cost effective.

7 Disadvantages of transdermal drug delivery:

- Transdermal drug delivery system cannot deliver ionic drugs.

- It cannot achieve high drug levels in blood.

- It cannot develop for drugs of large molecular size.

- It cannot deliver drugs in a pulsatile fashion.

- It cannot develop if drug or formulation causes irritation to skin.

- Possibility of local irritation at site of application.

- May cause allergic reaction.

- Sufficient aqueous and lipid solubility, a log $\mathrm{P}$ (octanol/ water) between 1 and 3 is required for permeate to transverse stratum corneum and underlying aqueous layer. 
- Only potent drugs are suitable candidates for transdermal patch because of the natural limits of drug entry imposed by the skin's impermeability.

- Long time adherence is difficult.

8 Kinetics of transdermal permeation: ${ }^{16}$

Skin permeation kinetics is important for the successful development of the transdermal systems. Transdermal permeation of a drug involves the following steps,

- Sorption by stratum corneum

- Penetration of drug through viable epidermis

- Uptake of the drug in the dermal papillary layer

The rate of permeation across the skin $(\mathrm{dQ} / \mathrm{dt})$ is given by, eqn 1

$$
\frac{\mathrm{dQ}}{\mathrm{dt}}=\mathbf{P}_{\mathrm{s}}\left(\mathbf{C}_{\mathrm{d}}-\mathbf{C}_{\mathbf{r}}\right)
$$

Where,

$\mathrm{P}_{\mathrm{s}}$ is the overall permeability constant of the skin tissue to the penetrant

$\mathrm{C}_{\mathrm{d}}$ is concentration of skin penetrant in the donor compartment (e.g., on the surface of stratum corneum)

$\mathrm{C}_{\mathrm{r}}$ is concentration in the receptor compartment (e.g., body)

$$
\mathbf{P}_{s}=\frac{\mathbf{K}_{s} \mathbf{D}_{s 5}}{\mathbf{h}_{s}}
$$

\section{$\ldots 2$}

Where,

$\mathrm{K}_{\mathrm{s}}$ is the partition coefficient for the interfacial partitioning of the penetrant molecule from a solution medium onto the stratum corneum $\mathrm{D}_{\mathrm{ss}}$ apparent diffusivity of the penetrant molecule $\mathrm{h}_{\mathrm{s}}$ overall thickness of skin tissues

As $\mathrm{K}_{\mathrm{s}}, \mathrm{D}_{\mathrm{ss}}$ and $\mathrm{h}_{\mathrm{s}}$ are constant under given conditions, the permeability coefficient $\left(\mathrm{P}_{\mathrm{s}}\right)$ for a skin penetrant can be considered to be constant.
From Eq.1 it is clear that a constant rate of drug permeation can be obtained only when $\left(C_{d}>C_{r}\right)$ i.e., the drug concentration at the surface of the stratum corneum $\left(\mathrm{C}_{\mathrm{d}}\right)$ is greater than the drug concentration in the body $\left(\mathrm{C}_{\mathrm{r}}\right)$ then Eq.1 becomes, eqn 3

$$
\frac{\mathrm{d} 0}{\mathrm{dt}}=\mathbf{P}_{\mathrm{s}} \mathbf{C}_{\mathrm{d}}
$$

And the rate of skin permeation is constant provided the magnitude of $\mathrm{C}_{\mathrm{d}}$ remains fairly constant throughout the course of skin permeation. For keeping $\mathrm{C}_{\mathrm{d}}$ constant the drug should be released from the device at a rate $R_{r}$ i.e. either constant or greater than the rate of skin uptake $R_{a}$ i.e. $\quad R_{r} \gg R_{a}$. Since $R_{r} \gg R_{a}$, the drug concentration on the skin surface $\mathrm{C}_{\mathrm{d}}$ is maintained at a level equal to or greater than the equilibrium solubility of the drug in the stratum corneum $\mathrm{C}_{\mathrm{s}}$ .i.e. $\mathrm{C}_{\mathrm{d}}>>\mathrm{C}_{\mathrm{s}}$. Therefore a maximum rate of skin permeation is obtained and is given by the equation, 4

$$
\left(\frac{\mathrm{dQ}}{\mathrm{dt}}\right)_{\mathrm{m}}=\mathbf{P}_{\mathrm{s}} \mathbf{C}_{\mathrm{s}}
$$

From the above equation it can be seen that the maximum rate of skin permeation depends upon the skin permeability coefficient $\mathrm{P}_{\mathrm{s}}$ and equilibrium solubility in the stratum corneum $\mathrm{C}_{\mathrm{s}}$. Thus skin permeation appears to be stratum corneum limited The membrane limited flux $(\mathrm{J})$ under steady state condition is described by equation, 5

$$
\mathbf{J}=\frac{\mathbf{D C K}_{0 / \mathrm{W}}}{\mathbf{h}}
$$

Where,

$\mathrm{J}$ is amount of drug passing through membrane system per unit area per unit time

$\mathrm{D}$ is diffusion coefficient of drug within the membrane

$\mathrm{C}$ is concentration gradient across the membrane

$\mathrm{K}$ is the membrane / vehicle partition coefficient $\mathrm{h}$ is the membrane thickness. 


\section{Factors affecting transdermal permeation Biological factor: ${ }^{14,20}$}

\subsection{Skin conditions:}

The intact skin itself acts as barrier but many agents like acids ,alkali cross the barrier cells and penetrates through the skin ,many solvents open the complex dense structure of horny layer Solvents like methanol, chloroform remove lipid fraction, forming artificial shunts through which drug molecules can pass easily.

\subsection{Skin age:}

It is seen that the skin of adults and young ones are more permeable than the older ones but there is no dramatic difference .Children shows toxic effects because of the greater surface area per unit body weight thus potent steroids, boric acid, hexachlorophene have produced severe side effects.

\subsection{Blood Supply:}

Changes in peripheral circulation can affect transdermal absorption.

\subsection{Regional skin site:}

Thickness of skin, nature of stratum corneum and density of appendages vary site to site. These factors affect significantly penetration.

\subsection{Skin metabolism:}

Skin metabolizes steroids, hormones, chemical carcinogens and some drugs. So skin metabolism determines efficacy of drug permeated through the skin.

\subsection{Species differences:}

The skin thickness, density of appendages and keratinization of skin vary species to species, so affects the penetration.

\section{Physicochemical factors: ${ }^{14}$ 10.1 Skin hydration:}

In contact with water the permeability of skin increases significantly. Hydration is most important factor increasing the permeation of skin. So use of humectant is done in transdermal delivery.

\subsection{Temperature and $\mathrm{pH}$ :}

The permeation of drug increases ten folds with temperature variation. The diffusion coefficient decreases as temperature falls. Weak acids and weak bases dissociate depending on the $\mathrm{pH}$ and $\mathrm{pKa}$ or $\mathrm{pKb}$ values. The proportion of unionized drug determines the drug concentration in skin. Thus, temperature and $\mathrm{pH}$ are important factors affecting drug penetration

\subsection{Diffusion coefficient:}

Penetration of drug depends on diffusion coefficient of drug. At a constant temperature the diffusion coefficient of drug depends on properties of drug, diffusion medium and interaction between them.

\subsection{Drug concentration:}

The flux is proportional to the concentration gradient across the barrier and concentration gradient will be higher if the concentration of drug will be more across the barrier.

\subsection{Partition coefficient:}

The optimal partition coefficient $(\mathrm{K})$ is required for good action. Drugs with high $\mathrm{K}$ are not ready to leave the ipid portion of skin. Also, drugs with low $\mathrm{K}$ will not be permeated.

\subsection{Molecular size and shape:}

Drug absorption is inversely related to molecular weight, small molecules penetrate faster than large ones.

\section{Environmental factors: ${ }^{20}$ \\ 11.1 Sunlight:}

Due to Sunlight the walls of blood vessels become thinner leading to bruising with only minor trauma in sun-exposed areas. Also pigmentation: The most noticeable sun-induced pigment change is a freckle or solar lentigo.

\subsection{Cold Season:}

Often result in itchy, dry skin. Skin responds by increasing oil production to compensate for the weather's drying effects. A good moisturizer will help ease symptoms of dry skin. Also, drinking lots of water can keep your skin hydrated and looking radiant.

\subsection{Air Pollution:}

Dust can clog pores and increase bacteria on the face and surface of skin, both of which lead to acne or spots. This affects drug delivery through the 
skin. Invisible chemical pollutants in the air can interfere with skin's natural protection system, breaking down the natural skin's oils that normally trap moisture in skin and keep it supple.

\subsection{Effect of Heat on Transdermal patch: ${ }^{22}$}

Heat induced high absorption of transdermal delivered drugs. Patient should be advised to avoid exposing the patch application site to external heat source like heated water bags, hot water bottles. Even high body temperature may also increase the transdermally delivered drugs. In this case the patch should be removed immediately.

Transdermal drug patches are stored in their original packing and keep in a cool, dry place until they are ready to used.

\section{Formulation of transdermal drug delivery system:}

Various components of a transdermal drug delivery system are shown in Fig.9.

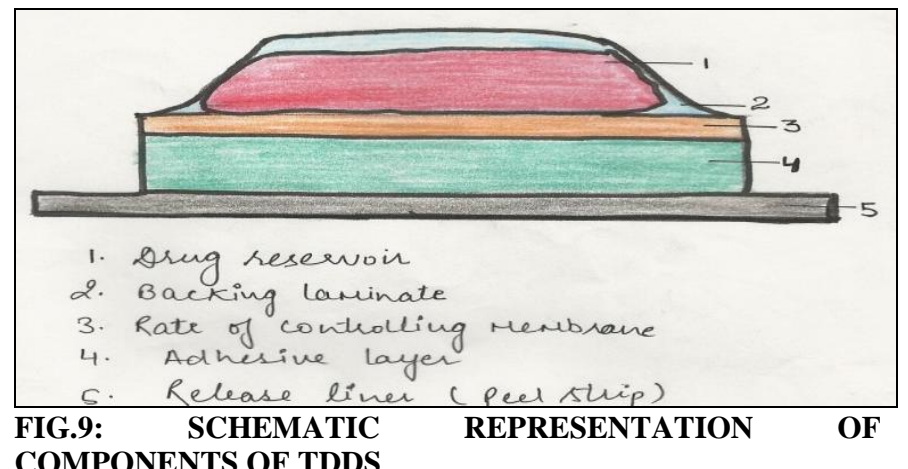

\subsection{Drug substance:}

For successfully developing a transdermal drug delivery system, the drug should be choosen with great care. The following are some of the desirable properties of a drug for transdermal delivery. ${ }^{28}$

\subsubsection{Physicochemical properties:}

- The drug should have a molecular weight less than 1000 Daltons.

- The drug should have affinity for both lipophilic and hydrophilic phasea. Extreme partitioning characteristics are not conductive to successful drug delivery via the skin.
- The drug should have low melting point.

- Along with these properties the drug should be potent, having short half life and be non-irritating.

\subsubsection{Biological Properties: ${ }^{1,17}$}

- Drug should be very potent ,i.e. it should be effective in few $\mathrm{mg}$ /day

- The drug should have short biological half life.

- The drug should not be irritant and non allergic to human skin.

- The drug should be stable when contact with the skin.

- They should not stimulate an immune reaction to the skin.

- Tolerance to the drug must not develop under near zero order release profile of transdermal delivery.

- Dose is less than $50 \mathrm{mg}$ per day, and ideally less than $10 \mathrm{mg}$ per day.

- The drug should not get irreversibly bound in the subcutaneous tissue.

- The drug should not get extensively metabolized in the skin.

Table 1 shows the important characteristics for an ideal candidate/ drug for TDD.

\begin{tabular}{cc} 
TABLE 1: IDEAL PROPERTIES OF & DRUG CANDIDATE \\
FOR TRANSDERMAL DRUG DELIVERY & \\
\hline Parameter & Properties \\
\hline Dose & Less than $20 \mathrm{mg} /$ day \\
Halflife & $<10 \mathrm{hrs}$ \\
Molecular weight & $<400$ Dalton \\
Melting point & $<200^{\circ} \mathrm{C}$ \\
Partition coefficient & 1 to 4 \\
Aqueous Solubility & $>1 \mathrm{mg} / \mathrm{mL}$ \\
pH of the aqueous saturated & $5-9$ \\
solution & $>0.5 \times 10^{-3} \mathrm{~cm} / \mathrm{h}$ \\
Skin Permeability Coefficient & Non irritating and non- \\
Skin Reaction & sensitizing \\
Oral Bioavailability & Low \\
\hline
\end{tabular}




\subsection{Polymer matrix:}

Polymers are the backbone of transdermal drug delivery system. System for transdermal delivery are fabricated as multi layered polymeric laminates in ehich a drug reservoir or a drug polymer matrix is sandwitched between two polymeric layers, an outer impervious backing layer that prevents the loss of drug through the backing surface and an inner polymeric layer that functions as an adhesive, or rate controlled membrane. ${ }^{25}$

\subsubsection{Ideal properties of a polymer to be used in a transdermal system:}

- Molecular weight, chemical functionality of the polymer should be such that the specific drug diffuses properly and gets released through it.
- The polymer should be stable.

- The polymer should be nontoxic

- The polymer should be easily of manufactured

- The polymer should be inexpensive

- The polymer and its deaggration product must be non toxic or non-antagonistic to the host.

- Large amounts of the active agent are incorporated into it.

Some commonly used polymer for TDD are shown in Table 2

TABLE 2: USEFUL POLYMERS FOR TRANSDERMAL DEVICES

\begin{tabular}{ccc}
\hline Natural Polymers & Synthetic Elastomers & Synthetic Polymers \\
\hline Cellulose derivatives & Polybutadiene & Polyvinylalcohol \\
Arabino Galactan & Hydrinrubber & Polyethylene \\
Zein & Polysiloxane & Polyviny Chloride \\
Gelatin & Acrylonitrile & Polyacrylates \\
Proteins & Neoprene & Polyamide \\
Shellac & Chloroprene & Acetal copolymer \\
Strarch & Silicon rubber & Polysyrene \\
\hline
\end{tabular}

\subsection{Penetration Enhancers:}

These are compounds which promote the skin permeability by altering the skin as barrier to the flux of a desired penetrate. ${ }^{16,26}$

\subsubsection{Ideal properties of penetration enhancers:}

- Controlled and reversible enhancing action

- Chemical and physical compatibility with drug and other pharmaceutical excipients

- Should not cause loss of body fluids, electrolytes or other endogenous materials
- Non toxic, non allergic, non irritating

- Pharmacological inertness

- Ability to act specifically for predictable duration

- Odorless, colorless, economical and cosmetically acceptable.

Some commonly used absorption enhancers for TDD shown in Table 3.

\section{TABLE 3: TYPES OF ABSORPTION ENHANCERS}

\begin{tabular}{cccc}
\hline Class & Examples & Mechanism & Transport Pathway \\
\hline Surfactants & Na-lauryl sulfate & Transcellular & Phospholipid acyl chain perturbation \\
& Polyoxyethylene-9-laurylether, & & Reduction mucus viscosity, Peptidase \\
& Bile salts: & Paracellular & inhibition \\
& Na-deoxycholate & & \\
Na-glycocholate & Na-taurocholate & Phospholipid acyl chain perturbation \\
\hline \multirow{2}{*}{ Fatty acids } & Oleic acid, & Transcellular & Phos \\
& & &
\end{tabular}




\begin{tabular}{cccc}
\hline Short fatty acids & Paracellular & \\
Cyclodextrins & $\begin{array}{c}\alpha-, \beta \text { - and } \gamma \text { cyclodextrins, } \\
\text { Methylated } \beta \text { cyclodextrins }\end{array}$ & Transcelular & Inclusion of membrane compounds \\
Chelating agents & $\begin{array}{c}\text { EDTA, } \\
\text { Polyacrylates }\end{array}$ & $\begin{array}{c}\text { Transcellular } \\
\text { Paracellular }\end{array}$ & $\begin{array}{c}\text { Complexation of } \mathrm{Ca}^{2+} \text { opening of tight } \\
\text { junctions }\end{array}$ \\
$\begin{array}{c}\text { Positively charged } \\
\text { polymer }\end{array}$ & $\begin{array}{c}\text { Chitosan salts, } \\
\text { Trimethyl chitosan }\end{array}$ & Paracellular & $\begin{array}{c}\text { Ionic interactions with negatively } \\
\text { charged groups of glycocalix }\end{array}$ \\
\hline
\end{tabular}

\subsection{Other excipients:}

Various solvents such as chloroform, methanol, acetone, isopropananol, and dichloromethane, are used to prepare drug reservoir. In addition plasticizers such as dibutylpthalate, propylene glycol are added to provide plasticity to the transdermal patch. ${ }^{26}$

\subsubsection{Pressure sensitive adhesive:}

A Pressure Sensitive Adhesive (PSA) is a material that helps in maintaining an intimate contact between transdermal system and the skin surface. It should adhere with not more than applied finger pressure, be aggressively and permanentaly tachy, exert a strong holding force. Additionally, it should be removable from the smooth surface without leaving a residue ${ }^{7}$ e.g.: polyacrylamates, polyacrylates, polyisobutylene, silicone based adhesive. The selection of an adhesive is based on numerous factors, including the patch design and drug formulation. PSA should be physicochemical and biologically compatible and should not alter drug release. The PSA can be positioned on the face of the device or in the back of the device and extending peripherally. ${ }^{30}$

\subsubsection{Backing laminates:}

While designing a backing layer the consideration of chemical resistance ${ }^{7}$ and excipients may compatible because the prolonged contact between the backing layer and the excipients, drug or penetration enhancer through the layer. They should a low moisture vapour transmission rate. They must have optimal elasticity, flexibility and tensile strength. eg: aluminium vapour coated layer, a plastic film and heat real layer. ${ }^{25}$

\section{Release linear:}

During storage release linear prevents the loss of drug that has migrated into the adhesive layer and contamination. ${ }^{25}$ However, as the linear is in intimate contact with the delivery system, it should comply with specific requirements regarding chemical inertness and permeation to the drug, penetration enhancer and water. ${ }^{7}$

\section{Major transdermal systems:}

13.1 Drug in adhesive system:

\subsubsection{Single layer drug in adhesive: ${ }^{40}$}

The adhesive layer of this system contains the drug. In this type of patch the adhesive layer not only serves to adhere the entire the various layers together, along with system to the skin, but is also responsible for releasing of the drug. ${ }^{17}$ The rate of release of drug from this type of system is dependent on the diffusion across the skin. ${ }^{27}$ The adhesive layer is surrounded by a temporary linear and a backing layer. ${ }^{8,}$ Shown in Fig. 10 $^{28}$

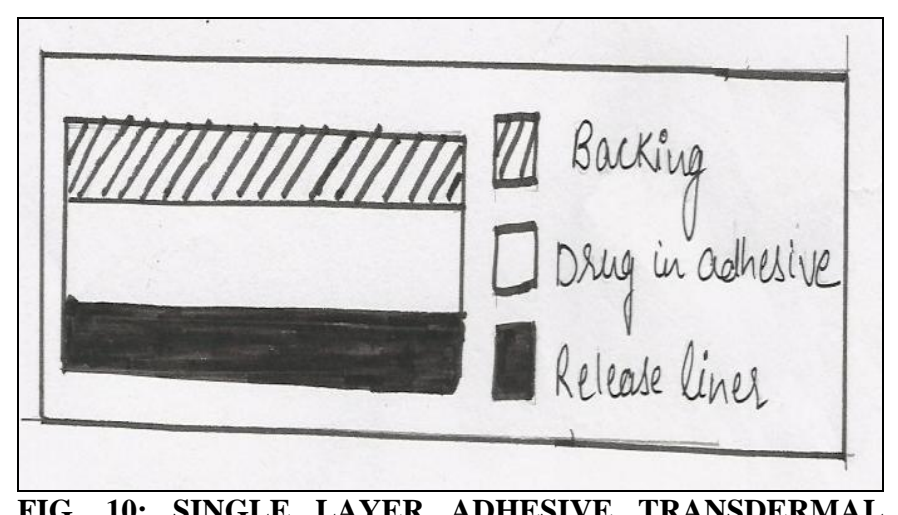

FIG. 10: SINGLE LAYER ADHESIVE TRANSDERMAL DELIVERY SYSTEM

\subsubsection{Multi layer drug in adhesion:}

The multi-layer drug-in adhesive patch is similar to the single-layer system in that both adhesive layers are also responsible for the releasing of the drug ${ }^{8}$ shown in Fig. 11. One of the layer is for immediate release of the drug and other layer is for control release of drug from the reservoir. The multi layer patch also has a temporary linear layer and a permanent backing. ${ }^{17}$ 


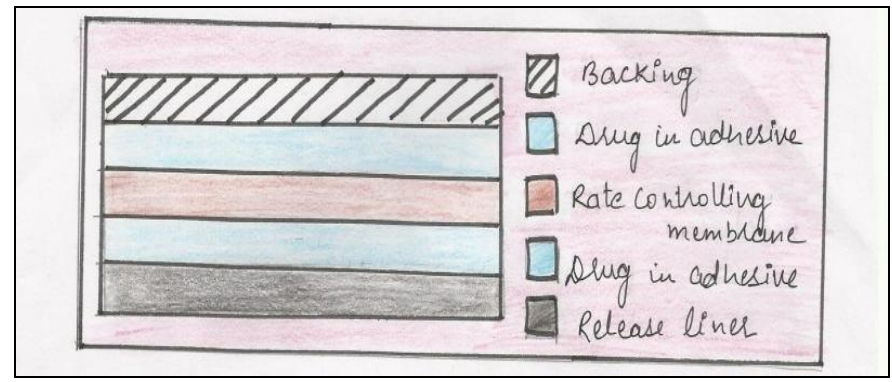

FIG. 11: MULTILAYERED DRUG IN ADHESIVE TRANSDERMAL SYSTEM.

\subsection{Reservoir:}

Unlike the single layer and multilayer drug in adhesive systems the reservoir transdermal system has a separate drug layer. ${ }^{17}$ The drug layer is a liquid compartment containing a drug solution or suspension separated by the adhesive layer. This patch is also backed by the backing layer, in Fig. $12{ }^{28}$ In this type of system the rate of release is zero order.

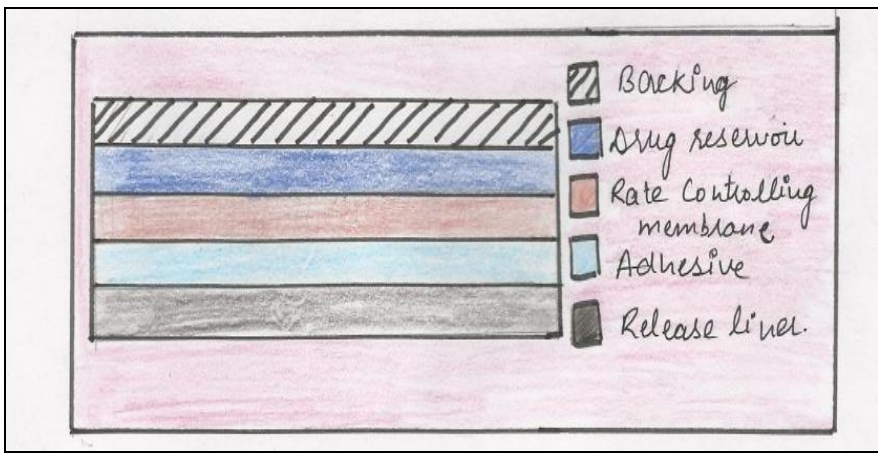

FIG.12: SCHEMATIC REPRESENTATION OF RESERVOIR TRANSDERMAL DELIVERY SYSTEM.

\subsection{Matrix:}

The Matrix system design as shown in Fig. 13 has a drug layer of a semisolid matrix containing a drug solution or suspension ${ }^{8}$. The adhesive layer in this patch surrounds the drug layer partially overlying it. These type of patches are also known as monolithic device. ${ }^{17}$

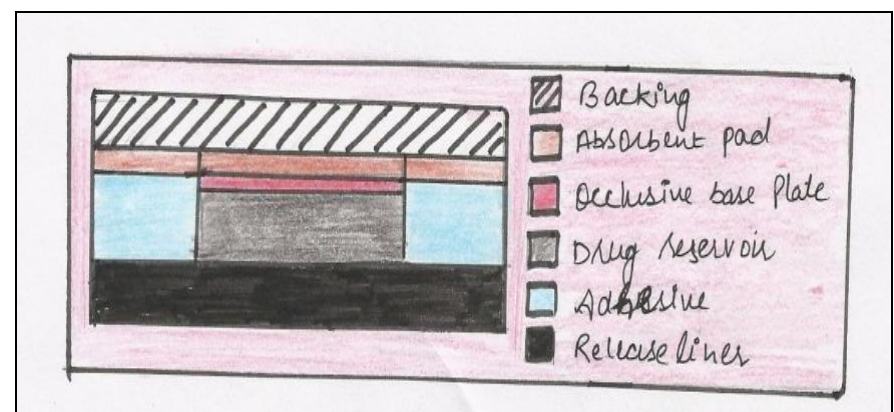

FIG. 13: SCHEMATIC REPRESENTATION OF MATRIX TRANSDERMAL DELIVERY SYSTEM

\subsection{Vapour Patch:}

In this type of patch the adhesive layer not only serves to adhere the various layers together but also to release vapour ${ }^{17}$. The vapour patches are new on the market and they release essential oils for up to 6 hours. The vapours patches release essential oils and are used in cases of decongestion mainly. Other vapour patches on the market are controlled vapour patches that improve the quality of sleep. ${ }^{20}$ Vapour patches that reduce the quantity of cigrate that one smokes in a mouth are also available on the market. ${ }^{8}$

14 Various methods for preparation of transdermal drug delivery system:

\subsection{Asymmetric TPX membrane method:}

A prototype patch can be fabricated by a heat sealable polyester film (type 1009, 3m) with a concave of $1 \mathrm{~cm}$ diameter used as the backing membrane. Drug sample is dispensed into the concave membrane, covered by a TPX \{poly (4methyl-1-pentene) $\}$ asymmetric membrane, and sealed by an adhesive. ${ }^{6}$

\subsubsection{Asymmetric TPX membrane preparation}

These are fabricated by using the dry/wet inversion process. TPX is dissolved in a mixture of solvent (cyclohexane) and nonsolvent additives at $60^{\circ} \mathrm{c}$ to form a polymer solution. The polymer solution is kept at $40^{\circ} \mathrm{C}$ for $24 \mathrm{hrs}$ and cast on a glass plate to a pre-determined thickness with a gardener knife. After that the casting film is evaporated at $50^{\circ} \mathrm{C}$ for $30 \mathrm{sec}$, then the glass plate is to be immersed immediately in coagulation bath [maintained the temperature at $25^{\circ} \mathrm{C}$ ]. After 10 minutes of immersion, the membrane can be removed, air dry in a circulation oven at $50^{\circ} \mathrm{C}$ for $\left.12 \mathrm{hrs}\right]^{3}$

\subsection{Circular teflon mould method: ${ }^{38}$}

Solutions containing polymers in various ratios are used in an organic solvent. Calculated amount of drug is dissolved in half the quantity of same organic solvent. Enhancers in different concentrations are dissolved in the other half of the organic solvent and then added. Di-Nbutylphthalate is added as a plasticizer into drug polymer solution. The total contents are to be stirred for $12 \mathrm{~h}$ and then poured into a circular teflon mould. The moulds are placed on a leveled surface and covered with an inverted funnel to 
control solvent vaporization in a laminar flow hood model with speed of air $1 / 2 \mathrm{~m} / \mathrm{sec}$. The solvent is allowed to evaporate for $24 \mathrm{~h}$. Before evaluation the dried films are to be stored for another $24 \mathrm{~h}$ at $25 \pm 0.5{ }^{\circ} \mathrm{C}$ in a desiccators containing silica gel before to eliminate aging effects. These types of films are to be evaluated within one week of their preparation. ${ }^{6}$

\subsection{Mercury substrate method:}

In this method drug is dissolved in polymer solution along with plasticizer. The above solution is to be stirred for $10-15 \mathrm{~min}$ to produce a homogeneous dispersion and poured in to a leveled mercury surface. Then the solution is covered with inverted funnel to control solvent evaporation. ${ }^{6}$

\subsection{By using "IPM membranes" method:}

In this method drug is dispersed in a mixture of water and propylene glycol containing carbomer 940polymer and stirred for $12 \mathrm{hrs}$ in magnetic stirrer. The dispersion is to be neutralized and made viscous by the addition of triethanolamine. Buffer $\mathrm{pH} 7.4$ can be used in order to obtain solution gel, if the drug solubility in aqueous solution is very poor. The formed gel will be incorporated in the IPM membrane. ${ }^{3}$

\subsection{By using "EVAC membranes" method:}

In order to prepare the target transdermal therapeutic system, 1\% carbopol reservoir gel, polyethelene (PE), ethylene vinyl acetate copolymer (EVAC) membranes can be used as rate control membranes. If the drug is not soluble in water, propylene glycol is used for the preparation of gel. Drug is dissolved in propylene glycol, carbopol resin will be added to the above solution and neutralized by using $5 \% \mathrm{w} / \mathrm{w}$ sodium hydroxide solution. The drug (in gel form) is placed on a sheet of backing layer covering the specified area. A rate controlling membrane will be placed over the gel and the edges will be sealed by heat to obtain a leak proof device. ${ }^{3}$

\subsection{Aluminium backed adhesive film method:}

Transdermal drug delivery system may produce unstable matrices if the loading dose is greater than $10 \mathrm{mg}$. Aluminium backed adhesive film method is a suitable one for preparation of same, chloroform is choice of solvent, because most of the drugs as well as adhesive are soluble in chloroform. The drug is dissolved in chloroform and adhesive material will be added to the drug solution and dissolved. A custammade aluminium former is lined with aluminium foil and the ends blanked off with tightly fitting cork blocks. ${ }^{3,6}$

\subsection{Preparation of TDDS by using proliposomes:}

The proliposomes are prepared by carrier method using film deposition technique. From the earlier reference drug and lecithin in the ratio of 1:2 can be used as an optimized ratio. The proliposomes are prepared by taking $5 \mathrm{mg}$ of mannitol powder in a $100 \mathrm{ml}$ round bottom flask which is kept at $60-70$ ${ }^{\circ} \mathrm{C}$ temperature and the flask is rotated at 80-90 $\mathrm{rpm}$ and dried the mannitol at vacuum for $30 \mathrm{~min}$. After drying, the temperature of the water bath is adjusted to $20-30{ }^{\circ} \mathrm{C}$. Drug and lecithin are dissolved in a suitable organic solvent mixture. Aliquot of $0.5 \mathrm{ml}$ of the organic solution is introduced into the round bottomed flask at $37{ }^{\circ} \mathrm{C}$ containing mannitol after complete drying second aliquots $(0.5 \mathrm{ml})$ of the solution is to be added. After the last loading, the flask containing proliposomes are connected in a lyophilizer and subsequently drug loaded mannitol powders (proliposomes) are placed in desiccators over night and then sieved through 100 mesh. The collected powder is transferred in to a glass bottle and stored at the freeze temperature until characterization. ${ }^{6}$

\subsection{By using free film method:}

Free film of cellulose acetate is prepared by casting on mercury surface. A polymer solution $2 \% \mathrm{w} / \mathrm{w}$ is prepared by using chloroform. Plasticizers are incorporated at a concentration of $40 \% \mathrm{w} / \mathrm{w}$ of polymer weight. Five $\mathrm{ml}$ of polymer solution was poured in a glass ring which is placed over the mercury surface in a glass petri dish. The rate of evaporation of the solvent is controlled by placing an inverted funnel over the petridish. The film formation is noted by observing the mercury surface after complete evaporation of the solvent. The dry film will be separated out and stored between the sheets of wax paper in desiccators until use. Free films of different thickness can be prepared by changing the volume of the polymer solution. ${ }^{6}$ 
15 Desirable features for transdermal patches: ${ }^{16}$

- Composition relatively invariant in use.

- $\quad$ System size reasonable.

- Defined site for application.

- Application technique highly reproducible.

- Delivery is zero order.

- Delivery is efficient.

\section{Conditions in which patches are used: ${ }^{8,20,21}$}

- When the patient has intolerable side effects (including constipation) and who is unable to take oral medication (dysphagia) and is requesting an alternative method of drug delivery.

- Where the pain control might be improved by reliable administration. This might be useful in patients with cognitive impairment or those who for other reasons are not able to selfmedicate with their analgesia.

- It can be used in combination with other enhancement strategies to produce synergistic effects.

\section{Conditions in which patches are not used: ${ }^{8}$, 20, 21, 37}

- Cure for acute pain is required.

- Where rapid dose titration is required.

- Where requirement of dose is equal to or less than $30 \mathrm{mg} / 24 \mathrm{hrs}$.

\section{Evaluation of transdermal patches:}

The transdermal patches can be characterized in terms of following parameters

- Physicochemical evaluation

- In vitro evaluation

- In vivo evaluation

\subsection{Physicochemical evaluation:}

Transdermal patches can be physicochemically evaluated in terms of these parameters:

\section{- Thickness:}

The thickness of transdermal film is determined by travelling microscope, dial gauge, screw gauge or micrometer at different points of the film. ${ }^{4}$

\section{- Uniformity of weight:}

Weight variation is studied by individually weighing 10 randomly selected patches and calculating the average weight. The individual weight should not deviate significantly from the average weight. $^{26,36}$

\section{- Drug content determination:}

An accurately weighed portion of film (about 100 $\mathrm{mg}$ ) is dissolved in $100 \mathrm{~mL}$ of suitable solvent in which drug is soluble and then the solution is shaken continuously for $24 \mathrm{~h}$ in shaker incubator. Then the whole solution is sonicated. After sonication and subsequent filtration, drug in solution is estimated spectrophotometrically by appropriate dilution. 8,39

\section{- Content uniformity test:}

10 patches are selected and content is determined for individual patches. If 9 out of 10 patches have content between $85 \%$ to $115 \%$ of the specified value and one has content not less than $75 \%$ to125\% of the specified value, then transdermal patches pass the test of content uniformity. But if 3 patches have content in the range of $75 \%$ to $125 \%$, then additional 20 patches are tested for drug content. If these 20 patches have range from $85 \%$ to $115 \%$, then the transdermal patches pass the test. $^{29}$

\section{- Moisture content:}

The prepared films are weighed individually and kept in a desiccators containing calcium chloride at room temperature for $24 \mathrm{~h}$. The films are weighed again after a specified interval until they show a constant weight. The percent moisture content is calculated using following formula.

$$
\% \text { Moisture content }=\text { Initial weight }- \text { Final weight X } 100^{4}
$$




\section{- Moisture Uptake:}

Weighed films are kept in a desiccator at room temperature for $24 \mathrm{~h}$. These are then taken out and exposed to $84 \%$ relative humidity using saturated solution of Potassium chloride in a desiccator until a constant weight is achieved. \% moisture uptake is calculated as given below. ${ }^{35}$

$\%$ moisture uptake $=$ Final weight - Initial weight X 100

\section{- Flatness:}

A transdermal patch should possess a smooth surface and should not constrict with time. This can be demonstrated with flatness study. For flatness determination, one strip is cut from the centre and two from each side of patches. The length of each strip is measured and variation in length is measured by determining percent constriction. Zero percent constriction is equivalent to 100 percent flatness.

$$
\% \text { constriction }=\mathrm{I} 1-\mathrm{I} 2 \mathrm{X} 100
$$

I2 = Final length of each strip

I1 = Initial length of each strip ${ }^{29}$

\section{- Folding Endurance:}

Evaluation of folding endurance involves determining the folding capacity of the films subjected to frequent extreme conditions of folding. Folding endurance is determined by repeatedly folding the film at the same place until it break. The number of times the films could be folded at the same place without breaking is folding endurance value. $^{31,41}$

\section{- Tensile Strength:}

To determine tensile strength, polymeric films are sandwiched separately by corked linear iron plates. One end of the films is kept fixed with the help of an iron screen and other end is connected to a freely movable thread over a pulley. The weights are added gradually to the pan attached with the hanging end of the thread. A pointer on the thread is used to measure the elongation of the film. The weight just sufficient to break the film is noted. ${ }^{26}$

\section{- Tack properties:}

It is the ability of the polymer to adhere to substrate with little contact pressure. Tack is dependent on molecular weight and composition of polymer as well as on the use of tackifying resins in polymer. ${ }^{30}$

\section{- Thumb tack test:}

The force required to remove thumb from adhesive is a measure of tack. ${ }^{31}$

\section{- Rolling ball test:}

This test involves measurement of the distance that stainless steel ball travels along an upward facing adhesive. The less tacky the adhesive, the further the ball will travel. ${ }^{30}$

\section{- Quick stick (Peel tack) test:}

The peel force required breaking the bond between an adhesive and substrate is measured by pulling the tape away from the substrate at 90 at the speed of $12 \mathrm{inch} / \mathrm{min}^{17}$

\section{- Probe tack test:}

Force required to pull a probe away from an adhesive at a fixed rate is recorded as tack. ${ }^{26}$

\subsection{In vitro release studies:}

Transdermal patches can be in vitro evaluated in terms of Franz diffusion cell the cell is composed of two compartments: donor and receptor. The receptor compartment has a volume of $5-12 \mathrm{ml}$ and effective surface area of 1-5 cm2. The diffusion buffer is continuously stirred at 600rpm by a magnetic bar. The temperature in the bulk of the solution is maintained by circulating thermostated water through a water jacket that surrounds the receptor compartment. The drug content is analyzed using suitable method, maintenance of sink condition is essential. ${ }^{33}$

\subsection{In vivo Studies:}

Transdermal patches can be in vivo evaluated in terms of In vivo evaluations are the true depiction of the drug performance. The variables which cannot be taken into account during in vitro studies can be fully explored during in vivo studies. In vivo evaluation of TDDS can be carried out using animal models human volunteers. ${ }^{26}$

\section{Animal models:}

Considerable time and resources are required to carry out human studies, so animal studies are preferred at small scale. The most common animal 
species used for evaluating transdermal drug delivery system are mouse, hairless rat, hairless dog, hairless rhesus monkey, rabbit, guinea pig etc. Various experiments conducted leads to a conclusion that hairless animals are preferred over hairy animals in both in vitro and in vivo experiments. Rhesus monkey is one of the most reliable models for in vivo evaluation of transdermal drug delivery in man. ${ }^{26}$

\section{Human model}

The final stage of the development of a transdermal device involves collection of pharmacokinetic and pharmacodynamic data following application of the patch to human volunteers. ${ }^{39}$ Clinical trials have been conducted to assess the efficacy, risk involved, side effects, patient compliance etc. Phase I clinical trials are conducted to determine mainly safety in volunteers and phase II clinical trials determine short term safety and mainly effectiveness in patients. Phase III trials indicate the safety and effectiveness in large number of patient population and phase IV trials at post marketing surveillance are done for marketed patches to detect adverse drug reactions. Though human studies require considerable resources best to assess the performance of the drug. ${ }^{26}$

\section{REFERENCES:}

a. Jalwal P, Jangra A, Dhaiya L, Sangwan Y, Saroha R. A review on transdermal patches. Pharm Res. J. 2010; 3:139149.

2. Bhowmik D, Chiranjib, Chandira M, Jayakar B, Sampath KP. Recent advances in transdermal drug delivery system. Int. J Pharm Tech Res. 2010; 2(1):68-77.

3. Kumar A, Pullankandam N, Prabhu SL, Gopal V. Transdermal drug delivery system: an overview. Int. J Pharm Sci. Review Res. 2010;3(2):49-54.

4. Divya A, Rao MK, Gnanprakash K, Sowjanya A, Vidyasagar N, Gobinath M. A review on current scenario of transdermal drug delivery system. Int. J Res. Pharm Sci. 2012;3(4):494-502.

5. Jain NK, Controlled and novel drug delivery. $1^{\text {st }}$ Ed., CBS Publisher and Distributors, New Delhi. 2001:100-129.

6. Rani S, Saroha K, Syan N, Mathur P. Transdermal patches a successful tool in transdermal drug delivery system. Plegia Res. Lib. 2011;2(5):17-29.

7. Dhawan S, Aggarwal G. Development, fabrication and evaluation of transdermal drug delivery system- a review. Pharm info.net. 2009:1-25.

8. Patel D, Chaudhary SA, Parmar B, Bhura N. Transdermal drug delivery system: a review. The Pharm Innovation. 2012;1(4):66-75.

9. Mehta R. Topical and transdermal drug delivery: what a pharmacist needs to know. InetCE. $1^{\text {st }}$ Ed., Arizona;2004:1-10.
10. Loyd V, Allen Jr, Nicholas G, Popovich, Howard C, Ansel. Pharmaceutical dosage forms and drug delivery systems, $8^{\text {th }}$ Edition., Wolter Kluwer Publishers, New Delhi;2005:298-299.

11. Jain NK. Pharmaceutical product development. $1^{\text {st }}$ Ed. CBS Publisher and Distributors. New Delhi. 2002:221228.

12. Robinson JR, Lee VH. Controlled drug delivery fundamentals and applications. $2^{\text {nd }}$ Ed. New York. 2005:523-536.

13. Wilson R, Waugh A, Grant A. Anatomy and physiology in health and illness. $9^{\text {th }}$ Ed. 2001 pg. 363-366.

14. Kumar D, Sharma N, Rana AC, Agarwal G, Bhat ZA. A review: transdermal drug delivery system: a tools for novel drug delivery sestem. Int. J Drug Dev. Res. 2011;3(3):7084.

15. Ramteke KH, Dhole SN, Patil SV. Transdermal drug delivery system: a review. J Advanced Sci. Res. 2012;3(1):22-35

16. Yadav V. Transdermal drug delivery system: review. Int. J Pharm Sci. Res. 2012;3(2):376-382.

17. Dhiman S, Thakur GS, Rehni AK. Transdermal patches: a recent approach to new drug delivery system. Int. J Pharmacy Pharm Sci. 2011;3(5):26-34.

18. Sharma RK, Keleb E, Mosa EB, Aljahwi AAZ. Transdermal drug delivery system- design and evaluation. Int. J Advances Pharm Sci. 2010;1:201-211.

19. Sandhu P, Bilandi A, Kataria S, Middha A. Transdermal drug delivery system (patches), applications in present scenario. Int. J Res. Pharm Chem. 2011;1(4):1139-1151.

20. Singh MC, Naik AS, Sawant SD. Transdermal drug delivery system with major emphasis on transdermal patches: a review. J Pharm Res. 2010;3(10):2537-2543.

21. Yadav B, Sharma B, Saroha K. Transdermal patches: a discrete dosage form. Int. J Current Pharm Res. 2011;3(3):98-108.

22. Tom WC. Effects of heat on transdermal drug patches. $1^{\text {st }}$ Ed.:1-2.

23. Kumar KPS, Bhowmik D, Chiranji BB, Chandira RM. Transdermal drug delivery system- a novel drug delivery system and its market scope and opportunities. Int. J Pharm Bio Sci. 2010.1(2):1-21.

24. Sharma RK, Keleb E, Mosa EB, Aljahwi AAZ. Transdermal drug delivery system- design and evaluation. Int. J Advances Pharm Sci. 2010;1:201-211.

25. Yadav B, Sharma B, Saroha K. Transdermal patches: a discrete dosage form. Int. $\mathbf{J}$ Current Pharm Res. 2011;3(3):98-108.

26. Gupta IK, Chokshi MM. Transdermal drug delivery system: an overview. Asian J Pharm Sci. Clinical Res. 2011;1(1):25-43.

27. Jatav VS, Saggu JS, Jat RK, Sharma AK, Singh RP. Recent advances in development of transdermal patches. Pharmacophore Int. Res. J. 2011;2(6):287-297.

28. Joshi K, Selvaduary G. Transdermal drug delivery system and their use of polymers. MatE 175- Biomaterials. $1^{\text {st }} \mathrm{Ed}$. 2008:1-28

29. Patel DS, Patel MV, Patel KN, Patel BA, Patel PA. Transdermal patches: a complete review on transdermal drug delivery system. Int. J Pharm Res. Scholars. 2012;1(1):55-71.

30. Lende LK, Grampurohit ND, Gaikwad DD, Gadhave MV, Jadhav SL. Transdermal patches: a review. Int. J Pharm Res. Dev. 2011;4(3):96-103.

31. Sakalle P, Dwivedi S, Dwivedi A. Design, evaluation, parameters and marketed products of transdermal patches: a review. J Pharm Res. 2010;3(2):235-240. 
32. Wokovich AM, Prodduturi S, Doub WH, Hussain AS, Buhse LF. Transdermal drug delivery system (TDDS) adhesion as a critical safety, efficacy and quality attribute. Eup. J Pharm BioPharm. 2006; 64:1-8.

33. Thejaswi C, Rao KM, Gobinath M, Radharani J, Hemafaith V, Venugopalaiah P. A review on design and characterization of proniosomes as a drug carrier. Int. J Advances Pharm Nanotechnology. 2011;1(1):16-19.

34. Arunachalam A, Karthikeyan M, Kumar DV, Pratap M, Sethuraman S, Kumar SA. Transdermal drug delivery system: review. Current Pharm Res. 2010;1(1):70-81.

35. Sachan B, Bajpai M. Transdermal drug delivery system: A review. Int. J Res. Dev. Pharm and life Sci. 2013;3(1):748765.

36. Bathe R, Kapoor R. Transdermal drug delivery system: formulation, development and evaluation-An overview. Int. J Biomedical and Advance Res 2015; 6(01):1-10
37. Patel D, Chaudhary A S, Parmar B. Transdermal drug delivery system: A review. The Pharm Innovation. 2012;1(4):66-75.

38. Shingade GM, Aamer Q, Sabale PM, Grampurohit ND, Gadhave MV, Jadhav SL, Gaikwad D. Review on: Recent Trends on Transdermal Drug Delivery System. J Drug Delivery Therapeutics 2012;2(1):66-75.

39. Sharma A, Saini S, Rana AC. Transdermal drug delivery system: A review. Int. J Res Pharm Biomedical Sci.2013;4(1):(286-292).

40. Mali AD, Bathe R, Patil M. An updated review on transdermal drug delivery systems. Int. J advances Sci. Res. 2015; 1(06): 244-254.

41. Gaikwad AK. Transdermal drug delivery system: Formulation aspects and evaluation. Comprehensive J Pharm Sci.2013;1(1):1-10.

\section{How to cite this article:}

Tanwar H and Sachdeva R: Transdermal Drug Delivery System: A Review. Int J Pharm Sci Res 2016; 7(6): 2274-90.doi: 10.13040/IJPSR.0975-8232.7(6).2274-90.

All @ 2013 are reserved by International Journal of Pharmaceutical Sciences and Research. This Journal licensed under a Creative Commons Attribution-NonCommercial-ShareAlike 3.0 Unported License.

This article can be downloaded to ANDROID OS based mobile. Scan QR Code using Code/Bar Scanner from your mobile. (Scanners are available on Google Playstore) 